\title{
Quality Assessments of Various Digital Image Fusion Techniques
}

\author{
D.A. Deshmukh ${ }^{1}$, Prof. Dr. P.V. Ingole ${ }^{2}$ \\ PG Scholar, Department of EXTC, PRMIT\&R, Badnera, Amravati, India ${ }^{1}$ \\ Professor, Department of EXTC, PRMIT\&R, Badnera, Amravati, India ${ }^{2}$
}

\begin{abstract}
Image Fusion is a process of combining the relevant information from a set of images into a single image, where the resultant fused image will be more informative and complete than any of the input images. The goal of image fusion (IF) is to integrate complementary multisensory, multitemporal and/or multiview information into one new image containing information the quality of which cannot be achieved otherwise. It has been found that the standard fusion methods perform well spatially but usually introduce spectral distortion, Image fusion techniques can improve the quality and increase the application of these data. In this paper we use various image fusion techniques using discrete wavelet transform and discrete cosine transform and it is proposed to analyze the fused image, after that by using various quality assessment factors it is proposed to analyze subject images and will draw a conclusion. In this results of several applications and comparisons between different fusion schemes and rules are addressed.
\end{abstract}

Keywords: image fusion, wavelet transform, discrete cosine transform, fused image.

\section{INTRODUCTION}

Nowadays, image fusion has become an important subarea of image processing. For one object or scene, multiple images can be taken from one or multiple sensors. These images usually contain complementary information. Image fusion is the process of detecting salient features in the source images and fusing these details to a synthetic image. Through image fusion, extended or enhanced information content can be obtained in the composite image, which has many application fields, such as digital imaging, medical imaging, remote sensing, and machine vision. The standard image fusion techniques, such as IHS based method, PCA based method and Brovey transform method operate under spatial domain. However, the spatial domain fusions may produce spectral degradation. This is particularly crucial in optical remote sensing if the images to fuse were not acquired at the same time. Therefore, compared with the ideal output of the fusion, these methods often produce poor result. Over the past decade, new approaches or improvements on the existing approaches are regularly being proposed to overcome the problems in the standard techniques. As multiresolution analysis has become one of the most promising methods in image processing, the wavelet transform has become a very useful tool for image fusion. It has been found that wavelet-based fusion techniques outperform the standard fusion techniques in spatial and spectral quality, especially in minimizing color distortion. Schemes that combine the standard methods (HIS or PCA) with wavelet transforms produce superior results than either standard methods or simple wavelet-based methods alone.

However, the tradeoff is higher complexity and cost. Image fusion has been used in many application areas. In remote sensing and in astronomy, multisensory fusion is used to achieve high spatial and spectral resolutions by combining images from two sensors, one of which has high spatial resolution and the other one high spectral resolution. Plenty of applications which use multisensor fusion of visible and infrared images have appeared in military, security, and surveillance areas. In the case of multiview fusion, a set of images of the same scene taken by the same sensor but from different viewpoints is fused to obtain an image with higher resolution than the sensor normally provides or to recover the $3 \mathrm{D}$ representation of the scene. The multitemporal approach recognizes two different aims. Images of the same scene are acquired at different times either to find and evaluate changes in the scene or to obtain a less degraded image of the scene.

The former aim is common in medical imaging, especially in change detection of organs and tumors, and in remote sensing for monitoring land or forest exploitation. The acquisition period is usually months or years. The latter aim requires the different measurements to be much closer to each other, typically in the scale of seconds, and possibly under different conditions. It also finds application in the area of navigation guidance, object detection and recognition. The present work offers a more extensive discussion of the approach and proposes a formalism as well as quality criteria

\section{LITERATURE SURVEY}

To study the concept of quality assessments of various digital image fusion techniques we have studied many papers. Here discussing some previous papers from which authors view and proposed method have been implemented. 
Vol. 6, Issue 1, January 2017

Richang Hong,Wenyi Cao,Jianxin Pang,Jianguo Jiang pixels in the process of similarity measure calculating. [1] has extended the work in image quality evaluation to a Experimental results demonstrate the superiority of our novel metric for objective evaluation of image fusion. The measure compared with conventional measures in terms of input images and the fused image are firstly converted into computation complexity and accuracy.

local sensitive intensity (LSI) by Radon transform. We then employ the sensitive intensity for measuring how much information have been transferred from each source into the fused result by the difference of LSI. All the LSI pairs are finally incorporated into the expression according to the Weber-Fechner law. Experimental results demonstrate that our proposed metric outperforms other metrics while it is consistent with the subjective evaluation. In this projection-based metric for the quantitative evaluation of pixel-level Image fusion is proposed. We project the input images onto the fused image's signal characterization to obtain project vector and measure the difference between the source images and the fused image by the projection vectors. Different from other quantitative evaluation methods, our proposed metric firstly introduce this type of methodology to evaluate the performance of image fusion by comparing the difference between the fused images and the source images. The experimental results demonstrated that this metric corresponds well to the subjective judgment and outperforms some other quantitative evaluation metrics.

Marcelino Anguiano-Morales, E. Noé Arias, G. Garnica, A. Martínez[2] has presented a method to extract shape and color information simultaneously of a colorful object by projecting sequentially sinusoidal fringe patterns onto object's surface. Distorted fringe patterns are captured by digital CCD color camera. It is applied the phase shifting method to evaluate the phase of the projected fringes. We obtain both topography details and color texture information. Also we introduced a three-step color phaseshifting method for measuring 3D shapes of color objects, using commercial CCD camera and DLP projector. Surface texture plays an important role in several engineering applications for shape analysis in industrial inspection, because it provides vital information of the object. This technique can be a great help to test the performance for the textures manufactured by machining processes. Although, our method may be impractical when capturing very unstable objects; this will be our next task. Implementing a high speed camera can be applied to dynamic events

Xiaoli Zhang, Xiongfei Li, Yuncong Feng[3] has presented a new image fusion performance measure, which consists of two parts: the first one is a similarity measure for predicting the amount of information transferred form original images to fused image in Riesz domains; the other is a measure for characterizing the contrast of the fused image. Considering that different morphological components share different importance in Human Visual System, a gradient based image content partition algorithm is adopted to segment original images and fused images into three parts, and according the partition results, different weights are given to different

Ming Yin,Wei Liua, Xia Zhaob, Yanjun Yinc, Yu Guoa[4] have proposed a novel sum-modified-Laplacian (NSML), which can extract more useful information from source images, is employed as the measurement to select bandpass sub-band coefficients. Finally, the fused image is obtained by performing the inverse NSST on the combined coefficients. The proposed fusion method is verified on several sets of multi-source images, and the experimental results show that the proposed approach can significantly outperform the conventional image fusion methods in terms of both objective evaluation criteria and visual quality for the band-pass sub-bands, we present a select rule by using a novel sum-modified-Laplacian. Several sets of experimental results show that the proposed method can capture the edge detail information of the source image and reduce the 'artificial information' and the impact of registration error, and improve the visual effect of the fused image

Alex Pappachen James,Belur V. Dasarathy [5] have provided a factual listing of methods and summarizes the broad scientific challenges faced in the field of medical image fusion. We characterize the medical image fusion research based on (1) the widely used image fusion methods, (2) imaging modalities, and (3) imaging of organs that are under study. This review concludes that even though there exists several open ended technological and scientific challenges, the fusion of medical images has proved to be useful for advancing the clinical reliability of using medical imaging for medical diagnostics and analysis, and is a scientific discipline that has the potential to significantly grow in the coming years.

Guang Zhu, Shu-Xu GuoSt[6] have proposed an active contour model (snake) based on image fusion, which is used in object detection and object tracking. Firstly, a multi-resolution image fusion with wavelet transform is applied to obtain the multi-resolution fused images. Secondly, in the low-frequency sub-image, a snake is applied with the Sobel operator to detect the object's contour; and in other high-frequency sub-images, a wavelet-based snake is applied.

The two convergent snakes are fused in a multi-resolution scheme to obtain a fused snake in the fused image in an original resolution. Experiment results indicate this fused snake's detection or tracking accuracy is improved greatly. Moreover, a snake fusion scheme is also devised based on a multi-resolution active contour with the Sobel operator and wavelet-based active contour, to improve the detection or tracking accuracy of our fused active contour. This fused multi-resolution active contour can be widely applied in object detection or tracking with complicated image backgrounds in the military or safety science. 
G. Piella, H. Heijmans[7] have presented three variants of a new quality metric for image fusion. The interest of our metrics, which are based on an image quality index recently introduced by Wang and Bovik in [Z. Wang et al., March 2002], lies in the fact that they do not require a ground-truth or reference image. We perform several simulations which show that our metrics are compliant with subjective evaluations and can therefore be used to compare different image fusion methods or to find the best parameters for a given fusion algorithm.

Guihong Qu, Dali Zhang and Pingfan Yan [8] have proposed an information measure for evaluating image fusion performance. The proposed measure represents how much information is obtained from the input images. No assumption is made regarding the nature of the relation between the intensities in both input modalities. The results show that the measure is meaningful and explicit. In this Letter they propose an information measure for evaluating the image fusion performance by using mutual information (MI). The proposed measure represents how much information obtained from the fusion of input images can be used to assess the performance of different image fusion algorithms. Since no assumption is made regarding the nature of the relation between the image intensities in the input images, the proposed measure is very general and can be applied automatically without prior processing.

Myungjin Choi, Rae Young Kim, Myeong-Ryong Nam, and Hong Oh Kim[9] have introduced an improved method of image fusion which is based on the amélioration de la resolution spatial par injection destructors (ARSIS) concept using the curvelet transform, because the curvelet transform represents edges better than wavelets. Because edges are fundamental in image representation, enhancing the edges is an effective means of enhancing spatial resolution.Curvelet-based image fusion has been used to merge a Land sat Enhanced Thematic Mapper plus Pan and MS image. The proposed method simultaneously provides richer information in the spatial and spectral domains. it represents edges better than a wavelet, the curvelet transform is well suited for the MSM in the ARSIS concept. Based on experimental results pertaining to seven indicators of Bias, SD, CC, RASE, ERGAS, , and sCC, the curvelet-based method provides better visual and quantitative results for remote sensing fusion.

M. Fallah Yakhdani, A. Azizi[10] have concentrated on the evaluation of theimage fusion techniques applied on the IRS P5 and P6 satellite images. The study area is chosen to cover different terrain morphologies. A good fusion scheme should preserve the spectral characteristics of the source multi-spectral image as well as the high spatial resolution characteristics of the source panchromatic image. In order to find out the fusion algorithm which is best suited for the P5 and P6 images, five fusion algorithms, such as Standard IHS, Modified IHS, PCA, Brovey and wavelet algorithms have been employed and analyzed. In this paper, eight evaluation criteria are also used for quantitative assessment of the fusion performance. The spectral quality of fused images is evaluated by the Spectral discrepancy, Correlation Coefficient (CC), RMSE and Mean Per Pixel Deviation (MPPD).For the spatial quality assessment, the Entropy, Edge detection, High pass filtering and Average Gradient(AG) are applied and the results are analyzed. The analysis indicates that the Modified IHS fusion scheme has the best definition as well as spectral fidelity, and has better performance with regard to the high textural information absorption. Therefore, as the study area is concerned, it is most suited for the IRS-P5 and P6 image fusion.

\section{III.PROPOSED SYSTEM}

In computer vision, Multisensor Image fusion is the process of combining relevant information from two or more images into a single image. The resulting image will be more informative than any of the input images.Multisensor data fusion has become a discipline which demands more general formal solutions to a number of application cases. Several situations in image processing require both high spatial and high spectral information in a single image. This is important in remote sensing.

However, the instruments are not capable of providing such information either by design or because of observational constraints. One possible solution for this is data fusion.

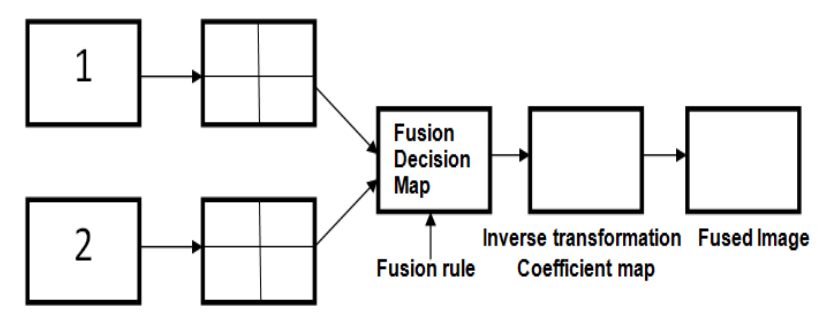

Source images Transformation coefficient maps

Fig:- Block diagram of a generic image fusion approach

Transformation coefficient is first performed on each source images, and then a fusion decision map is generated based on a set of fusion rules. The fused transformed coefficient map can be constructed from the wavelet coefficients of the source images according to the fusion decision map. Finally the fused image is obtained by performing the inverse wavelet transform. From the above diagram, we can see that the fusion rules are playing a very important role during the fusion process.

A) Discrete cosine transforms (DCT):-

A discrete cosine transform (DCT) expresses a finite sequence of data points in terms of a sum of cosine functions oscillating at different frequencies. DCTs are important to numerous applications in science and 
Vol. 6, Issue 1, January 2017

engineering, from lossy compression of audio (e.g. MP3) and images (e.g. JPEG) (where small high-frequency components can be discarded), to spectral methods for the numerical solution of partial differential equations. The use of cosine rather than sine functions is critical for compression, since it turns out (as described below) that fewer cosine functions are needed to approximate a typical signal, whereas for differential equations the cosines express a particular choice of boundary conditions. The most common variant of discrete cosine transform is the type-II DCT, which is often called simply "the DCT"..$^{[1][2]}$ Its inverse, the type-III DCT, is correspondingly often called simply "the inverse DCT" or "the IDCT". Two related transforms are the discrete sine transforms (DST), which is equivalent to a DFT of real and odd functions, and the modified discrete cosine transforms (MDCT), which is based on a DCT of overlapping data The DCT, and in particular the DCT-II, is often used in signal and image processing, especially for lossy compression, because it has a strong "energy compaction" property.

\section{B) Discrete wavelet transforms (DWT):-}

The discrete wavelet transform (DWT) is an implementation of the wavelet transform using a discrete set of the wavelet scales and translations obeying some defined rules. In other words, this transform decomposes the signal into mutually orthogonal set of wavelets, which is the main difference from the continuous wavelet transform (CWT), or its implementation for the discrete time series sometimes called discrete-time continuous wavelet transform (DT-CWT). The wavelet can be constructed from a scaling function which describes its scaling properties. The restriction that the scaling functions must be orthogonal to its discrete translations implies some mathematical conditions on them which are mentioned everywhere. In numerical analysis and functional analysis, a discrete wavelet transform (DWT) is any wavelet transform for which the wavelets are discretely sampled. As with other wavelet transforms, a key advantage it has over Fourier transforms is temporal resolution: it captures both frequency and location information (location in time).

\section{IV.CONCLUSION}

Image Fusion is a process of combining the relevant information from a set of images into a single image, where the resultant fused image will be more informative and complete than any of the input images. Image fusion techniques can improve the quality and increase the application of these data.

In this paper by using transformation coefficient based fusion technique for the image fusion we will analyze the fused image, after that by using various quality assessment factors we can analyze this image and draw a conclusion that from which transformation technique we can find the better results. In this several applications and comparisons between different fusion schemes and rules will addressed.

\section{ACKNOWLEDGMENT}

It is indeed a matter of great privilege to publish this paper on "Quality Assessments of Various Digital Image Fusion Techniques " under the valuable guidance of Prof. Dr. P.V. Ingole. We would like to express our deep sense of gratitude to our guide for this valuable guidance, advice and constant work.

\section{REFERENCES}

[1] Richang Hong,Wenyi Cao,Jianxin Pang,Jianguo Jiang," Directional projection based image fusion quality metric" Information Sciences 281 (2014) 611-619

[2] Marcelino Anguiano-Morales, E. Noé Arias, G. Garnica, A. Martínez," Image fusion by color texture extraction" Optik 125 (2014) 810-812K. Elissa, "Title of paper if known," unpublished.

[3] Xiaoli Zhang, Xiongfei Li, Yuncong Feng," A new image fusion performance measure using Riesz transforms" Optik 125 (2014) $1427-1433$

[4] Ming Yin,Wei Liua, Xia Zhaob, Yanjun Yinc, Yu Guoa," A novel image fusion algorithm based on nonsubsampled shearlet transform" Optik 125 (2014) 2274-2282M. Young, The Technical Writer's Handbook. Mill Valley, CA: University Science, 1989.

[5] Alex Pappachen James,Belur V. Dasarathy," Medical image fusion: A survey of the state of the art" Information Fusion 19 (2014) 4-19

[6] Guang Zhu, Shu-Xu GuoSt," Image-fusion-based multi-resolution active contour modelGuang"'Optik 125 (2014) 4955-495

[7] G. Piella ,H. Heijmans,"A new quality metric for image fusion" Image Processing, 2003. ICIP 2003. Proceedings. 2003 International Conference

[8] Guihong Qu, Dali Zhang and Pingfan Yan,'Information measure for performance of image fusion" ELECTRONICS LETTERS 28th March 2002 Vol. 38 No. 7

[9] Myungjin Choi, Rae Young Kim, Myeong-Ryong Nam, and Hong Oh Kim," Fusion of Multispectral and Panchromatic Satellite Images Using the Curvelet Transform" IEEE Geoscience and Remote Sensing Letters ( Volume: 2,Issue:2,April2005)

[10] M. Fallah Yakhdani, A. Azizi,"Quality assessment of image fusion techniques for multi sensor high resolution satellite images" Centre of Excellence for Natural Disaster Management, Department of Geomatics Engineering, College of Engineering, University of Tehran, Iran - (mfallah84@gmail.com, aazizi@ut.ac.ir) Commission VII, WG VII/6 